\title{
Adherence to systemic therapies for immune- mediated inflammatory diseases in Lebanon: a physicians' survey from three medical specialties
}

This article was published in the following Dove Press journal:

Patient Preference and Adherence

15 May 2017

Number of times this article has been viewed

\author{
Alfred Ammoury' \\ Jad Okais² \\ Mireille Hobeika ${ }^{3}$ \\ Raymond B Sayegh ${ }^{4}$ \\ Rani H Shayto 5 \\ Ala I Sharara ${ }^{5}$ \\ 'Division of Dermatology, St George \\ Hospital University Medical \\ Center, ${ }^{2}$ Division of Rheumatology, \\ St Joseph University, ${ }^{3} \mathrm{AbbVie}$ Levant, \\ ${ }^{4}$ Division of Gastroenterology, \\ St Joseph University, ${ }^{5}$ Department \\ of Internal Medicine, Division \\ of Gastroenterology, American \\ University of Beirut Medical Center, \\ Beirut, Lebanon
}

Background: Immune-mediated inflammatory diseases (IMIDs) are chronic conditions that may cause tissue damage and disability, reduced quality of life and increased mortality. Various treatments have been developed for IMIDs, including immune modulators and targeted biologic agents. However, adherence remains suboptimal.

Methods: An adherence survey was used to evaluate physicians' beliefs about adherence to medication in IMID and to evaluate if and how they manage adherence. The survey was distributed to 100 randomly selected physicians from three different specialties. Results were analyzed by four academic experts commissioned to develop an action plan to address practical and perceptual barriers to adherence, integrating it into treatment goals to maximize outcomes in IMID, thereby elevating local standards of care.

Results: Eighty-two physicians participated in this study and completed the questionnaire. Most defined adherence as compliance with prescribed treatment. Although the majority of surveyed physicians (74\%) did not systematically measure adherence in their practice, 54\% identified adherence as a treatment goal of equal or greater importance to therapeutic endpoints. Lack of time and specialized nursing support was reported as an important barrier to measuring adherence. The expert panel identified four key areas for action: $360^{\circ}$ education (patient-nursephysician), patient-physician communication, patient perception and concerns, and market access/cost. An action plan was developed centered on education and awareness, enhanced benefit-risk communication, development of adherence assessment tools and promotion of patient support programs.

Conclusion: Nonadherence to medication is a commonly underestimated problem with important consequences. A customized target-based strategy to address the root causes of nonadherence is essential in the management of chronic immune-mediated diseases.

Keywords: biologics, compliance, Crohn's disease, psoriasis, rheumatoid arthritis

\section{Introduction}

Immune-mediated inflammatory diseases (IMIDs), including Crohn's disease, psoriasis and rheumatoid arthritis (RA), are chronic conditions affecting millions of individuals worldwide, with a prevalence of $5 \%-7 \%$ in the western society. ${ }^{1}$ Untreated or inadequately treated IMIDs naturally progress, causing severe tissue damage and disability, reduced quality of life and increased mortality. ${ }^{1,2}$ In addition, IMIDs are associated with emergency room visits, hospitalizations and surgeries, all of which substantially elevate health care costs. ${ }^{1,3}$ Various treatments have been developed for IMIDs, including topical agents, systemic corticosteroids, immunosuppressants and biologic agents. ${ }^{4}$ Recent clinical trials have provided strong evidence that biologic
Correspondence: Ala I Sharara

Department of Internal Medicine,

Division of Gastroenterology, American University of Beirut Medical Center, PO Box II-0236/16-B, Beirut, Lebanon Tel +96 I I 350000 ext 535 I

Fax +96| | 366098

Email ala.sharara@aub.edu.lb 
agents are safe and highly effective in reducing inflammation and preventing associated tissue damage in patients affected by IMIDs. ${ }^{4}$ However, though biologics have revolutionized the treatment of IMIDs, $30 \%-40 \%$ of patients still do not respond to therapy. The reasons for this are complex and include, among others, alternative inflammatory pathways to the one targeted by the specific biologic, development of neutralizing antidrug antibodies and pharmacodynamic or pharmacokinetic reasons. ${ }^{4}$ Notably, another important reason for "nonresponse" is related to lack of adherence to therapy. As true for all medications that target chronic diseases, it has become clear that adherence to systemic therapies including biologics remains suboptimal. The burden of the problem is often underestimated and not fully appreciated in its full extent. Poor medication adherence presents a significant global problem; a 2003 World Health Organization (WHO) report found that global medication adherence among patients with chronic diseases averages 50\%, and that the impact of poor adherence increases as the proportion of patients with chronic diseases increases worldwide. The report also notes that nonadherence is a leading cause of preventable morbidity, mortality and cost. ${ }^{5,6}$ A recent meta-analysis ${ }^{7}$ including 98 studies with $>200,000$ patients confirms these findings and reports that the discontinuation rates of tumor necrosis factor (TNF) inhibitors at 4 years were up to $52 \%$. The reasons for this are multidimensional, including, as expected, lack of treatment efficacy (therapy-related factors), but other factors may also be at play. The WHO recognizes the relevance of four other categories affecting medication use, including socioeconomic, patient-related, conditionrelated and health care team/system factors. ${ }^{5,6}$

In the latter category, patients' surveys have consistently identified the relationship with physicians and health care providers (HCP) as a fundamental element in their adherence to therapeutic regimens. By the same token, the awareness and attitude of physicians and HCP to this problem is critical to its solution. Physicians/HCP awareness and attitude to this problem and their consequent ability to influence patients' adherence varies considerably according to personal, cultural, societal and the local health care system Therefore, as there is a link between adherence and disease outcomes, to improve adherence, it is important also to gauge physicians' and HCP attitude in the context of the specific socioeconomic environment and health care system they operate in.

It is with this in mind that we conducted a physicians' survey on nonadherence to systemic therapies among Lebanese experts from three different specialties (dermatology, gastroenterology and rheumatology) and from three different academic institutions (St George Hospital University Medical Center, St Joseph University and the American University of Beirut) and community specialists, in an effort to understand physicians' perceptions of adherence and identify possible specific factors for nonadherence among Lebanese IMID patients. Better understanding of such factors would contribute to the development of local strategies for increasing patient adherence to these medications and for improving health care provision and, most importantly, patients' outcomes.

\section{Methods}

An adherence survey developed by Abbott Global Team to evaluate the views and opinions of physicians and HCP regarding adherence to medication in IMIDs was conducted using pen and paper. The survey was developed by Abbott Global Team and then reviewed and implemented by the medical team in Lebanon. The survey was used to evaluate beliefs of Lebanese HCP specialists about adherence with different types of medication and their level of knowledge regarding factors affecting adherence. There was written notice on all the surveys stating clearly that the survey results may be analyzed and published. This survey was voluntary and the physicians' participation implied consent. Surveyed physicians were informed that the information collected was confidential and contained no personal identifiers. The survey included questions exploring physicians' beliefs, for example, "Do you think there is room for improvement in patients' adherence to treatment in IMIDs?" and "How well do you think you are managing adherence in your patients?", as well as questions aiming at capturing the physician's definition of adherence, for example, "How would you define adherence to medication?". Finally, the survey addressed questions related to potential operational and organizational barriers and directly evaluated whether adherence was actually measured at the workplace and who was responsible for the management of patient adherence, for example, educating, counseling and assessing adherence. Thus, the survey was administered to capture and evaluate beliefs of Lebanese HCP specialists about adherence with different types of medication and evaluate their level of knowledge regarding factors affecting adherence. The objective was also to evaluate if and how adherence was managed in their local practice.

The survey was distributed to 100 randomly selected physicians from three different specialties (dermatology, rheumatology and gastroenterology) from different Institutions as well as private clinics in Lebanon. These included 
25 rheumatologists, 37 gastroenterologists and 38 dermatologists. The demographic data of the participants were as follows: the majority were males, only three were females, the average age was 40 years, while the average number of years in practice was 8 years. To avoid potential bias related to financial inducements, no compensation was offered to participants. Surveys were collected 1 month after delivery. Physicians who failed to respond after 1 month were sent reminders. The data were analyzed using descriptive statistics. The study was approved by the Institutional Research Committee of the St Georges Hospital University Medical Center, which deemed the research exempt from participant written consent.

\section{Results}

Eighty-two physicians returned the completed survey. They consisted of 22 rheumatologists, 30 gastroenterologists and 30 dermatologists. The reasons that may influence adherence to medication as perceived by the physicians, according to their definition of adherence, are summarized in Figure 1. Most physicians defined adherence as compliance and/or taking all doses or $>75 \%$ of prescribed medications. Also, $25 \%$ and $17 \%$ thought that sticking to regular dose and taking all medications regularly would facilitate adherence, while $<10 \%$ felt that frequency, duration and amount of drug, low treatment cost, doing blood tests and patient education were less important.

Factors affecting adherence are summarized in Figure 2. As expected, fear of treatment side effects and relationship with doctors, as well as depression, treatment expectations and disease duration were all reported as of major importance (over 30\%). Likewise, ease of drug administration, disease knowledge and relationship with nurse scored highly $(28 \%$, $28 \%$ and $23 \%$, respectively), whereas disease severity did not score $>27 \%$. Age and gender scored $19 \%$ and $18 \%$, respectively, while smoking scored only $7 \%$.

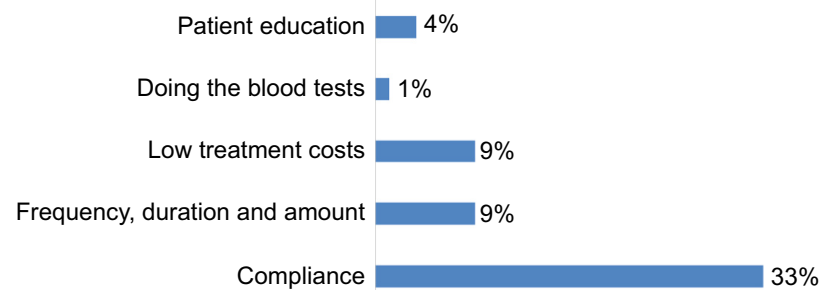

Taking all medications regularly $\quad 17 \%$

Sticking to regular dose $>75 \%$ - $25 \%$

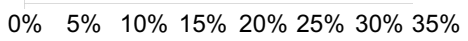

Figure I Definition of adherence according to surveyed physicians.

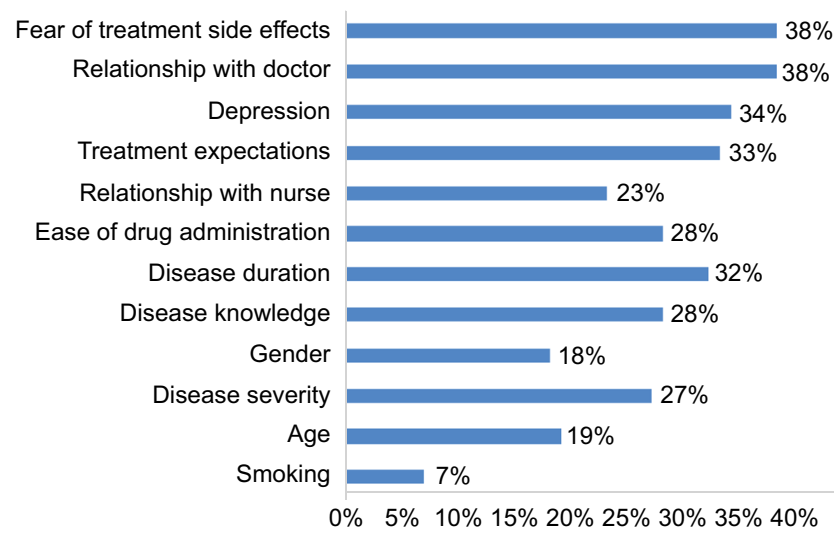

Figure 2 Identified factors influencing adherence.

The results representing the pooled responses by physicians from the three medical specialties (dermatology, rheumatology and gastroenterology) are illustrated in Figure 3. No differences were noted across specialties. The workplace distribution was widely represented, including hospital and academic settings as well as outpatients and specialist clinics. Eighty-one percent thought that there is room for improvement in patients' adherence to treatment. While 39\% thought that adherence to treatment is as important as other therapeutic goals and, importantly, most physicians $(60 \%)$ believed that the management of patient adherence is their responsibility. However, notably, only $26 \%$ reported actually measuring adherence, while nearly half of the physicians (44\%) believed they are managing adherence adequately.

\section{Discussion}

Though the treatment of IMIDs has been revolutionized by the advent of biologic therapies with major improvements in disease outcomes and impact on patients' ability to return to a productive life, $30 \%-40 \%$ of patients still do not respond to therapy. The reasons for this are multiple, but adherence to treatment is becoming increasingly recognized as an important factor. For example, a recent meta-analysis ${ }^{7}$ in RA including 98 studies with $>200,000$ patients confirms that the discontinuation rates of TNF inhibitors at 4 years were up to $52 \%$. Though the most frequent cause for discontinuation remains therapy inefficacy and/or adverse events, nonadherence also plays a major part in poor disease control and outcomes.

In inflammatory bowel disease (IBD), nonadherence results in an increased risk of disease recurrence, colorectal cancer, hospitalization and medical services costs. $^{6-9}$ Although there are limited data on the impact of 


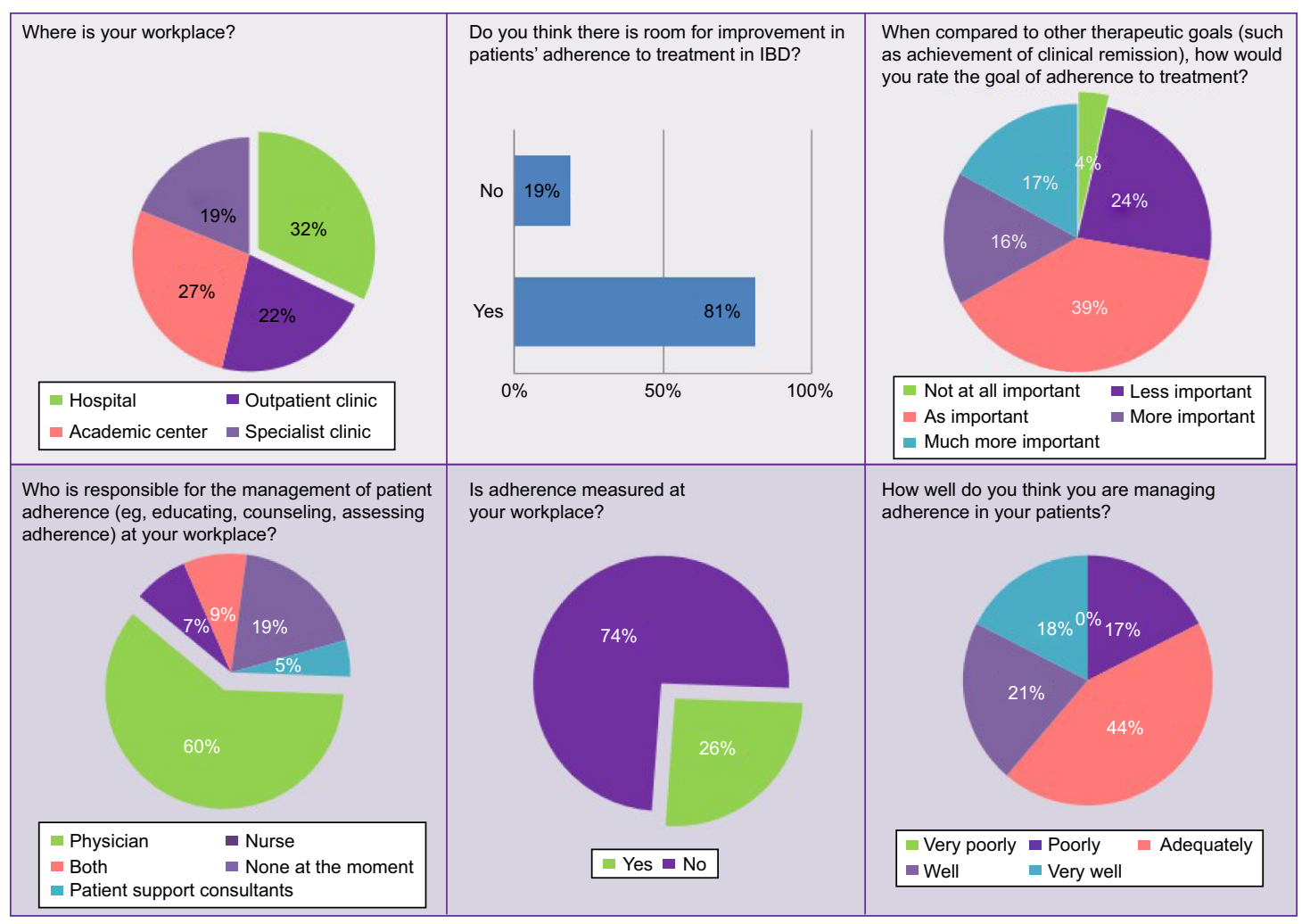

Figure 3 Survey combined results.

Abbreviation: IBD, inflammatory bowel disease.

nonadherence in psoriasis, ${ }^{10,11}$ higher health care utilization and lower quality of life have been reported. Likewise, nonadherence in RA has important clinical outcomes, including increased disease activity and flares, lower rates of sustained remission, and patient-reported outcomes. ${ }^{12-14}$

The $\mathrm{WHO}^{6}$ has identified five main category factors that affect medication-taking behavior, which physicians/ HCP and patients must both recognize. These include social and economic factors, health care team and system-related factors, condition-related factors, therapy-related factors and patient-related factors. These are interrelated, and a multidisciplinary effort is often required in order to improve many of these aspects in an attempt to improve medication adherence both in the general population and in specific IMID cases. Notably, factors such as time spent with the doctor, continuity of care, communication style and interpersonal style of the physician have been reported to be superior to sociodemographic factors in affecting medication adherence. ${ }^{5}$ Salt and Frazier reported that increased patient knowledge about RA and satisfaction with HCP interactions had improved medication adherence. ${ }^{15}$ Similarly, in a study investigating RA patients' satisfaction with the health care they are receiving, Kjeken et a $\mathrm{l}^{16}$ found that patients who were highly involved in their own care were more likely to be satisfied
(91\% vs $61 \%$ in the low involvement group), ${ }^{17}$ stressing the importance of patient involvement and a positive physicianpatient effort as a driver of better medication adherence.

Health care team and system-related factors have not been sufficiently investigated with respect to patient medication adherence. While a healthy physician-patient relationship may improve adherence, poorly developed health services, poor medication distribution methods, lack of knowledge and training in managing chronic illnesses, weak capacity to educate patients about adherence ${ }^{6}$ and other factors may impact adherence. By developing greater awareness of causes and predictors of nonadherence, health care professionals will be better prepared to identify patients at risk of nonadherence and, consequently, poised to develop appropriate patientspecific interventions to improve adherence.

It is in this context that this survey was conducted and the results show they are in line with the published literature and support the concept that improving physicians' and HCP's awareness of patients' beliefs and factors that influence adherence can impact on disease responsiveness and outcomes. The results of the survey also support the development of adherence-improving methods including patient education and improved physician-patient communication. In particular, given the chronicity of IMID and the need for 
maintenance therapy, a strong physician-patient relationship is essential to promote adequate education and expectations in the context of the local cultural and health care system.

The experience in Lebanon has solidified the concept that patient care should be a multidisciplinary approach where patients are managed on a case-by-case basis. Alongside providing appropriate medical care, the survey documents that physicians acknowledge the patients' role in the decisionmaking process through understanding their beliefs of their disease process and tailoring a customized patient-centered intervention plan. Physician-patient communication must be improved in an effort to accommodate patient autonomy and partnership in the decision-making process as well as support self-efficacy. Adopting a multidisciplinary approach can improve the quality of medication-taking behavior of patients. Furthermore, the survey has emphasized that there is a need for ongoing education at different levels and it should not be assumed that patients understand "simple" statistical concepts and that physicians and HCP should communicate the risks clearly with the use of diagrams or examples to keep things in perspective. In addition, it would be important to steer patients away from unregulated websites and instead point them to expert-recommended patient websites. The key to all of these principles is that interventions cannot be successful without continuous collection and use of feedback from the patients targeted by the intervention. As a result of the survey, key challenges for optimal adherence in the Lebanese population were identified and an experts' meeting was called to develop an action plan aimed at addressing them; they are summarized in Table 1.

From this it is clear that limited education is an important contributor to nonadherence, not only at the patient level, but also at the level of HCP. A solution to this problem would be empowering already existing patients' advocacy groups, which can initiate awareness campaigns that can in turn enhance patients' knowledge about disease processes and the importance of adherence to treatment. Nurses' and physicians' training programs can be implemented in institutions and hospitals, which will also raise awareness about patients' current adherence behavior, how to better investigate adherence patterns and how to encourage and promote better medication adherence behaviors. One of the observed problems with the Lebanese current health care system was the less-than-optimal physician-patient relationship. Most physicians have extremely busy schedules, preventing sufficient time from being dedicated to patient encounters. Some physicians also adopt the notion of paternalistic medicine, where patients are not disclosed all the details concerning their disease processes and care plan. Thus, it is important to move away from paternalistic practices to a more balanced physician-patient partnership where disease management is shared between patients and physicians in order to improve treatment acceptance, compliance and adherence (both compliance and persistence). Physicians have the duty to address patients' beliefs about medication regimens, answer questions and recognize fears hindering treatment. Physicians, nurses, pharmaceutical companies and other health care professionals are urged to invest in patient education materials, such as visual tools and brochures, to aid in translating difficult and complex medical concepts into more comprehensible material at the patients' disposal.

The lack of proper assessment of adherence is another obstacle identified in our study, notably adherence to therapy was measured only in $26 \%$ of workplaces. Most physicians take patient adherence for granted and may not suspect nonadherence as a cause of treatment failure. This is likely to lead to decisions such as escalating drug doses or switching medications without addressing the root cause of nonadherence. Thus, it would be recommended that a standardized method

Table I Key challenges and actions for treatment adherence

\begin{tabular}{|c|c|}
\hline Key challenges & Key actions \\
\hline \multirow[t]{2}{*}{ Limited education at three levels (patient-physician-nurse) } & Empower patient advocacy groups \\
\hline & Nurse training programs within institutions/hospitals \\
\hline Suboptimal physician-patient relation/communication & $\begin{array}{l}\text { Shared decision making between patients and physicians to improve acceptance, } \\
\text { compliance and persistence }\end{array}$ \\
\hline Physicians beliefs & Develop and support IMID interest group through respective medical societies \\
\hline \multirow[t]{2}{*}{ Patients' beliefs/concerns about therapy } & Develop and distribute patient educational material and simple visual tools \\
\hline & Improve communication with physician and enhance nursing support and access \\
\hline \multirow[t]{2}{*}{ Lack of assessment of adherence/assessment tools } & Development of a simple questionnaire to identify major gaps leading to nonadherence \\
\hline & Encourage related psychological and behavioral research \\
\hline Market access/reimbursement issues & Patient advocacy groups to lobby third-party payers \\
\hline Unintentional nonadherence & $\begin{array}{l}\text { Reminders, mobile applications with push notifications, partner and/or family } \\
\text { involvement }\end{array}$ \\
\hline
\end{tabular}

Abbreviation: IMID, immune-mediated inflammatory diseases. 
of measuring medication adherence with an acceptable level of accuracy such as patient questionnaires, pill counts or other methods as noted in the literature is implemented in routine management. Market access and monetary reimbursement also contribute to patient nonadherence. Based on this study, it is recommended that patient advocacy groups should lobby third-party payers in order to make effective medications available for all patients irrespective of socioeconomic status. Unintentional nonadherence was another reason observed from our model, where patients forget to take medication at designated time, dosage and frequency. Unintentional nonadherence can be addressed by creating mobile applications or other forms of reminders, as well as by family education and support in order to ensure appropriate medication-taking behavior and improve adherence.

\section{Conclusion}

Left untreated or inadequately treated, IMIDs can cause severe disability, greatly impaired quality of life and even increase the risk of premature death. Newly developed biologic agents offer effective and generally safe treatment options for individuals affected by IMIDs such as psoriasis, RA and Crohn's disease or ulcerative colitis. However, there is substantial reason to believe that some patients miss the full benefit of biologic treatments because of nonadherence. The consequences are likely to mirror the increased morbidity, mortality, health care costs and productivity losses observed among patients with poor adherence. The results of this survey reinforce the importance of basic and well-tested principles that can be used to develop adherence interventions customized for specific patient populations using systemic therapies in the context of specific health care systems and cultural and social environments.

\section{Acknowledgments}

This study was supported by an educational grant from AbbVie.

A preliminary abstract of this paper was presented at the American College of Gastroenterology Annual Scientific Conference as a poster presentation. The poster's abstract was published in "Poster Abstracts" as: Sharara AI, Ammoury A, Okais J, Sayegh R. Physicians' perspective regarding adherence to systemic therapies for IBD and other immunemediated inflammatory diseases. Am J Gastroenterol. 2014;109(Suppl 2s):S512. A1729.

\section{Disclosure}

The authors report no conflicts of interest in this work.

\section{References}

1. El-Gabalawy H, Guenther LC, Bernstein CN. Epidemiology of immunemediated inflammatory diseases: incidence, prevalence, natural history, and comorbidities. J Rheumatol Suppl. 2010;85:2-10.

2. Kuek A, Hazleman BL, Ostor AJ. Immune-mediated inflammatory diseases (IMIDs) and biologic therapy: a medical revolution. Postgrad Med J. 2007;83(978):251-260.

3. Jacobs P, Bissonnette R, Guenther LC. Socioeconomic burden of immune-mediated inflammatory diseases-focusing on work productivity and disability. $J$ Rheumatol Suppl. 2011;88:55-61.

4. Smolen JS, Aletaha D. Rheumatoid arthritis therapy reappraisal: strategies, opportunities and challenges. Nat Rev Rheumatol. 2015; 11(5):276-289.

5. World Health Organization. Adherence to Long-Term Therapies: Evidence for Action; 2003. Available from: http://www.who.int/ chp/knowledge/publications/adherence_full_report.pdf. Accessed December 8, 2012.

6. Kane S, Huo D, Aikens J, Hanauer S. Medication nonadherence and the outcomes of patients with quiescent ulcerative colitis. Am J Med. 2003;114(1):39-43.

7. Souto A, Maneiro JR, Gómez-Reino JJ. Rate of discontinuation and drug survival of biologic therapies in rheumatoid arthritis: a systematic review and meta-analysis of drug registries and health care databases. Rheumatology (Oxford). 2016;55(3):523-534.

8. van Staa TP, Card T, Logan RF, Leufkens HG. 5-Aminosalicylate use and colorectal cancer risk in inflammatory bowel disease: a large epidemiological study. Gut. 2005;54(11):1573-1578.

9. Kane SV, Chao J, Mulani PM. Adherence to infliximab maintenance therapy and health care utilization and costs by Crohn's disease patients. Adv Ther. 2009;26(10):936-946.

10. Bhosle MJ, Feldman SR, Camacho FT, Timothy Whitmire J, Nahata MC, Balkrishnan R. Medication adherence and health care costs associated with biologics in Medicaid-enrolled patients with psoriasis. J Dermatolog Treat. 2006;17(5):294-301.

11. Zaghloul SS, Goodfield MJ. Objective assessment of compliance with psoriasis treatment. Arch Dermatol. 2004;140(4):408-414.

12. Pascual-Ramos V, Contreras-Yanez I, Villa AR, Cabiedes J, Rull-Gabayet M. Medication persistence over 2 years of follow-up in a cohort of early rheumatoid arthritis patients: associated factors and relationship with disease activity and with disability. Arthritis Res Ther. 2009;11(1):R26.

13. Contreras-Yanez I, Cabiedes J, Villa AR, Rull-Gabayet M, Pascual-Ramos V. Persistence on therapy is a major determinant of patient-, physician- and laboratory-reported outcomes in recentonset rheumatoid arthritis patients. Clin Exp Rheumatol. 2010;28(5): 748-751.

14. Contreras-Yanez I, Ponce De Leon S, Cabiedes J, Rull-Gabayet M, Pascual-Ramos V. Inadequate therapy behavior is associated to disease flares in patients with rheumatoid arthritis who have achieved remission with disease-modifying antirheumatic drugs. Am J Med Sci. 2010;340(4):282-290.

15. Salt E, Frazier SK. Adherence to disease-modifying antirheumatic drugs in patients with rheumatoid arthritis: a narrative review of the literature. Orthop Nurs. 2010;29(4):260-275.

16. Kjeken I, Dagfinrud H, Mowinckel P, Uhlig T, Kvien TK, Finset A. Rheumatology Care: Involvement in medical decisions, received information, satisfaction with care, and unmet health care needs in patients with rheumatoid arthritis and ankylosing spondylitis. Arthritis Rheum. 2006;55(3):394-401.

17. Barton JL. Patient preferences and satisfaction in the treatment of rheumatoid arthritis with biologic therapy. Patient Prefer Adherence. 2009;3:335-344. 
Patient Preference and Adherence

Dovepress

\section{Publish your work in this journal}

Patient Preference and Adherence is an international, peer-reviewed, open access journal that focuses on the growing importance of patient preference and adherence throughout the therapeutic continuum. Patient satisfaction, acceptability, quality of life, compliance, persistence and their role in developing new therapeutic modalities and compounds to optimize

Submit your manuscript here: http://www.dovepress.com/patient-preference-and-adherence-journ clinical outcomes for existing disease states are major areas of interest for the journal. This journal has been accepted for indexing on PubMed Central The manuscript management system is completely online and includes a very quick and fair peer-review system, which is all easy to use. Visit http://www. dovepress.com/testimonials.php to read real quotes from published authors. 